\title{
Comparison of Oculus Rift and HTC Vive: Feasibility for Virtual Reality-Based Exploration, Navigation, Exergaming, and Rehabilitation
}

\author{
Adrián Borrego, BSc, Jorge Latorre, BSc, Mariano Alcañiz, PhD, and Roberto Llorens, PhD ${ }^{1,2}$
}

\begin{abstract}
Introduction: The latest generation of head-mounted displays (HMDs) provides built-in head tracking, which enables estimating position in a room-size setting. This feature allows users to explore, navigate, and move within real-size virtual environments, such as kitchens, supermarket aisles, or streets. Previously, these actions were commonly facilitated by external peripherals and interaction metaphors.

Objective: The objective of this study was to compare the Oculus Rift and the HTC Vive in terms of the working range of the head tracking and the working area, accuracy, and jitter in a room-size environment, and to determine their feasibility for serious games, rehabilitation, and health-related applications.

Materials and Methods: The position of the HMDs was registered in a $10 \times 10$ grid covering an area of $25 \mathrm{~m}^{2}$ at sitting $(1.3 \mathrm{~m})$ and standing $(1.7 \mathrm{~m})$ heights. Accuracy and jitter were estimated from positional data. The working range was estimated by moving the HMDs away from the cameras until no data were obtained.

Results: The HTC Vive provided a working area $\left(24.87 \mathrm{~m}^{2}\right)$ twice as large as that of the Oculus Rift. Both devices showed excellent and comparable performance at sitting height (accuracy up to $1 \mathrm{~cm}$ and jitter $<0.35 \mathrm{~mm}$ ), and the HTC Vive presented worse but still excellent accuracy and jitter at standing height (accuracy up to $1.5 \mathrm{~cm}$ and jitter $<0.5 \mathrm{~mm}$ ). The HTC Vive presented a larger working range $(7 \mathrm{~m})$ than did the Oculus Rift (4.25 m).

Conclusion: Our results support the use of these devices for real navigation, exploration, exergaming, and motor rehabilitation in virtual reality environments.
\end{abstract}

Keywords: Virtual reality, Head-mounted display, Serious games, Rehabilitation, Exergaming, Navigation

\section{Introduction}

$\mathbf{V}$ IRTUAL REALITY (VR) is defined as the simulation of an environment or activity through the real-time stimulation of one or more sensory channels, thus allowing for real-time user interaction. ${ }^{1}$ Since VR's very beginnings, its feasibility and concept have been linked to existing technologies for stimulation and interaction. Actually, the characteristics of the technology have been shown to modulate immersion, the extent to which VR is capable of delivering an illusion of reality to a human participant's senses. ${ }^{2}$ This immersion, in turn, affects presence, the sense of being in the virtual environment. ${ }^{2,3}$ Given the importance of sight in human perception, ${ }^{4}$ anatomically evidenced by the large number of brain areas involved in visual processing and cross-modal association, ${ }^{5}$ most development efforts have focused on improving visual stimulation. Traditional VR settings involve projected display screens that completely surround the viewer, known as Cave automatic virtual environment or CAVE systems ${ }^{6}$ or display screens fixed to the head near the eyes that completely substitute real-world input with computer-generated media, known as head-mounted displays (HMDs). ${ }^{7}$

The technology-mediated simulation of enriched ecologically valid environments and the provision of controlled sensory stimulation are especially interesting for serious games, rehabilitation, and health-related applications. ${ }^{1}$ However, adapting VR for rehabilitation has revealed some weaknesses, such as the side effects of display characteristics, as well as obstacles, such as the cost/benefit of the technology, that prevent its adoption in most rehabilitation centers. ${ }^{8}$

\footnotetext{
${ }^{1}$ Neurorehabilitation and Brain Research Group, Instituto de Investigación e Innovación en Bioingeniería, Universitat Politècnica de València, Valencia, Spain.

${ }^{2}$ Servicio de Neurorrehabilitación y Daño Cerebral de los Hospitales NISA, Fundación Hospitales NISA, Valencia, Spain.
} 

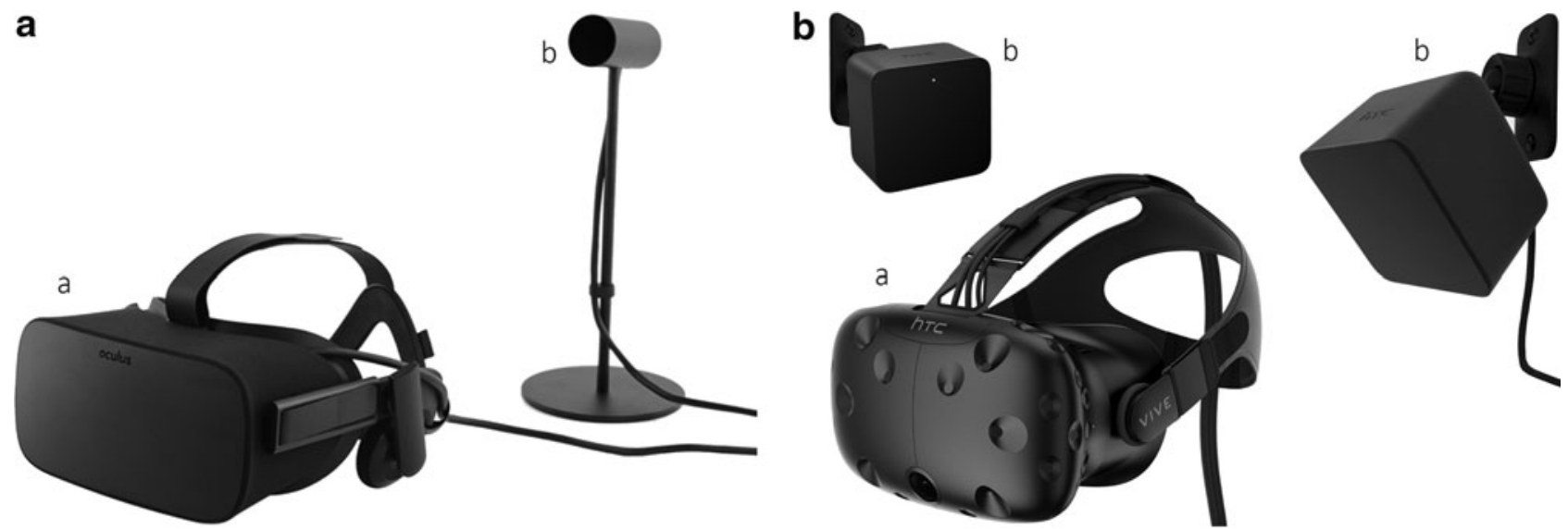

FIG. 1. Two last-generation head-mounted displays, the (a) Oculus Rift and (b) the HTC Vive, were analyzed in this study. Both devices consisted of a display (a) and a tracking module (b).

Concurrent with the renewed interest in $\mathrm{VR},{ }^{9}$ an increasing number of systems have been presented in the past years. ${ }^{10-13}$ As described by the manufacturers, these new devices provide improved features, such as higher resolution, faster refresh rate, and wider field of view, at dramatically lower costs than early systems. In addition, new HMDs include built-in tracking mechanisms to estimate the position and orientation of the head in real time, which enables perspective correction and allows users to freely move, at least to some extent, as in the real world in a room-size environment. From the clinical point of view, the possibility to move and walk as in the real world could maximize the ecological validity of virtual environments and tasks that require users to move, such as walking and navigating simulations or exergaming and serious games for motor rehabilitation. ${ }^{14-17}$ These tasks could also increase skill transference from the virtual to the real world. ${ }^{18}$ Although the HMDs' tracking features might be determinant for these applications, their characteristics and feasibility still remain unknown.

The objective of this study was threefold: (1) to compare the working area, accuracy, and jitter of the head tracking of two of the most renown last generation HMDs, the Oculus Rift Consumer Version 1 (Oculus VR, Irvine, CA) and the HTC Vive (HTC Corporation, Taoyuan City, Taiwan) in a room-size setting; (2) to determine the working range of both devices; and (3) to model the dependence of the accuracy of the head tracking systems with distance.

\section{Materials and Methods}

\section{Instrumentation}

This study involved both the Oculus Rift and the HTC Vive (Fig. 1). The characteristics of both headsets have been provided by their manufacturers (Table 1) and can also be

Table 1. Comparison of the Specifications of Oculus Rift and HTC Vive

\begin{tabular}{|c|c|c|}
\hline & Oculus Rift & HTC Vive \\
\hline Display & OLED & OLED \\
\hline Display size & $90 \mathrm{~mm} \times 2456 \mathrm{ppi}$ & $91.9 \mathrm{~mm} \times 2447 \mathrm{ppi}$ \\
\hline Resolution & $2160 \times 1200$ pixels & $2160 \times 1200$ pixels \\
\hline Refresh rate & $90 \mathrm{~Hz}$ & $90 \mathrm{~Hz}$ \\
\hline \multicolumn{3}{|l|}{ Field of view } \\
\hline Horizontal & $94^{\circ}$ & $110^{\circ}$ \\
\hline Vertical & $93^{\circ}$ & $113^{\circ}$ \\
\hline Lens type & Fresnel & Fresnel \\
\hline Lens adjustment & $\begin{array}{l}\text { IPD (58-72 mm), lens-to-eye distance } \\
\text { (adjustable with optional glasses spacer) }\end{array}$ & $\begin{array}{l}\text { IPD (60.8-74.6 mm), lens-to-eye distance } \\
\text { ("eye-relief" adjustment) }\end{array}$ \\
\hline Sensors & Accelerometer, gyroscope, magnetometer & Accelerometer, gyroscope \\
\hline Integrated camera & No & Yes \\
\hline Audio & $\begin{array}{l}\text { Microphone, integrated supra-aural } \\
\text { 3D spatial audio headphones (removable) }\end{array}$ & Microphone, jack for external headphones \\
\hline Wireless & Bluetooth & Bluetooth \\
\hline Ports & Proprietary headset connector (HDMI/USB 3.0) & HDMI 1.4, USB $3.0 \times 2$ \\
\hline Materials used & Plastic, IR-transparent fabric, glass, foam rubber & Plastic, glass, foam rubber \\
\hline $\begin{array}{l}\text { Weight } \\
\quad \text { (excluding cable) }\end{array}$ & $470 \mathrm{~g}$ & $563 \mathrm{~g}$ \\
\hline
\end{tabular}

The table describes the characteristics of the head-mounted displays used in the study. 
found elsewhere. ${ }^{19}$ Regarding tracking, the Oculus Rift uses a microphone-shaped IR camera designed to be fixed on a conventional table and tilted toward the HMD. This IR camera locates a constellation of IR LEDs that are embedded in the headset but not directly visible (Fig. 1a). The HTC Vive, in contrast, uses two base stations designed to be fixed on a wall above head height, ideally more than $2 \mathrm{~m}$. Each base station must be placed facing the other at a maximum distance of $5 \mathrm{~m}$, and also tilted toward the HMD (Fig. 1b). Similar to lighthouses, the base stations include two stationary LED arrays that flash at $60 \mathrm{~Hz}$ and two active spinning laser emitters that throw a beam of light after each flash. The headset has a constellation of photodiodes that estimate the series of flashes. The position and orientation of the headset can be estimated from the delay between the flash emitted by the LED array and that emitted by the spinning emitter in each photodiode.

A VR-ready high-end computer, equipped with an 8-core Intel $^{\circledR}$ Core $^{\mathrm{TM}}$ i7-6700K @ 4.00 GHz, 16 GB of RAM, and a NVIDIA $^{\circledR}$ Geforce $^{\circledR}$ GTX 980 with 12 GB of GDDR5, was used with both HMDs during the experiment. The 1.10.0 and 1.1.1 versions of the tracking plugin for Unity were used for the Oculus Rift and the HTC Vive, respectively.

\section{Procedure}

To estimate the working area, accuracy, and jitter of the tracking of both systems in a room-size setting, a $10 \times 10$ grid with $50 \times 50 \mathrm{~cm}$ squares was marked on the floor covering an area of $25 \mathrm{~m}^{2}$. The Oculus tracking camera was fixed at a height of $70 \mathrm{~cm}$, centered along the $x$ axis, at $x=2.5 \mathrm{~m}$, $y=-0.75 \mathrm{~m}$ (Fig. 2a). The HTC Vive base stations were placed on a lateral of the grid at a height of $250 \mathrm{~cm}$, at positions $x=1.5 \mathrm{~m}, y=3.0 \mathrm{~m}$ and $x=5.0 \mathrm{~m}, y=-0.5 \mathrm{~m}$, respectively, at the maximum distance recommended by the manufacturer ( $5 \mathrm{~m}$ ) (Fig. 2b). Each was oriented to the other and tilted $40^{\circ}$. The HMDs were sequentially placed in all the grid's intersection and border points (121 points in total) at 1.7 and $1.3 \mathrm{~m}$, the approximate heights of a subject's head in the standing and sitting positions, respectively, using a spe- cially designed device that prevented movement and allowed height adjustment. ${ }^{17,20}$ For the points where tracking data were available, the positions of both HMDs, always oriented in the $-\hat{y}$ direction (Fig. 2), were registered for 5 seconds at $50 \mathrm{~Hz}$.

To estimate the working range of both systems' tracking, a measuring tape was fixed on the floor along the longitudinal axis of the Oculus Rift's tracking camera and a base station of the HTC Vive. Following the same procedure previously described, the positions of both HMDs, always oriented to the tracking camera or base station, were registered for 5 seconds at 1.3 and $1.7 \mathrm{~m}$ every $25 \mathrm{~cm}$, moving away until no tracking data were obtained.

\section{Data analysis}

At each point of the grid, accuracy $(e)$ was estimated as the mean difference between the position of the HMD measured in the real world and its estimated position. Jitter $(j)$ was calculated as the standard deviation in the estimated position in the time interval as follows ${ }^{17}$ :

$$
e=\frac{1}{N} \sum_{i=1}^{N}\left|X_{i}-\tilde{X}_{i}\right|
$$

$$
j=\sqrt{\sum_{i=1}^{N} \frac{X_{i}^{2}}{N}-\bar{X}^{2}}
$$

where $N$ is 250 , the number of measurements provided by the tracking system for 5 seconds at $50 \mathrm{~Hz} ; X_{i}$ is the real position of the headset, estimated as the distance from the origin of coordinates to the HMD in the real world; $\tilde{X}_{i}$ is the estimated position of the headset provided by the Unity plugin; and $\bar{X}$ is the mean position during these 5 seconds. The mean and standard deviation for all points were estimated for both accuracy and jitter. Data were separately analyzed inside and outside the working area recommended by the manufacturers: for the Oculus Rift, a circle of radius
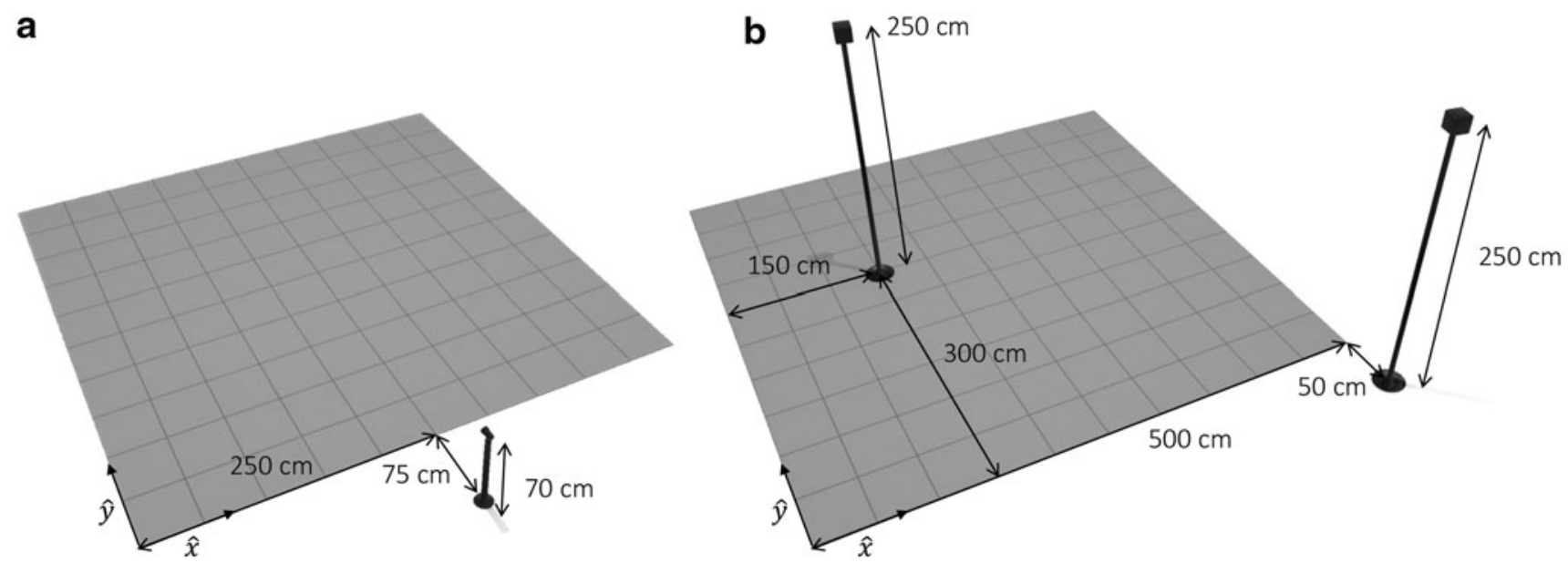

FIG. 2. A $10 \times 10$ grid with $50 \times 50 \mathrm{~cm}$ squares was marked on the floor. (a) The Oculus tracking camera was fixed at a height of $70 \mathrm{~cm}$, centered along the $x$ axis, at $x=2.5 \mathrm{~m}, y=-0.75 \mathrm{~m}$. (b) The base stations of the HTC Vive were placed on a lateral of the grid at a height of $250 \mathrm{~cm}$, at positions $x=1.5 \mathrm{~m}, y=3.0 \mathrm{~m}$ and $x=5.0 \mathrm{~m}, y=-0.5 \mathrm{~m}$, respectively, one oriented to the other and tilted $40^{\circ}$, at the maximum distance recommended by the manufacturer $(5 \mathrm{~m})$. 
$50 \mathrm{~cm}$ was centered in the longitudinal axis of the camera at $1.25 \mathrm{~m}$ away; for the HTC Vive, the inner area of the rectangle was defined by the base stations.

All analyses were computed with MATLAB Release 2013b (The MathWorks, Inc., Natick, MA).

\section{Results}

The experimental results showed a working area of $11.75 \mathrm{~m}^{2}$ for the Oculus Rift and $24.87 \mathrm{~m}^{2}$ for the HTC Vive at all heights, which greatly exceeded the maximum recommended working areas of 2.75 and $6.25 \mathrm{~m}^{2}$ for both devices, respectively. Figure 3 depicts the working areas of both devices.

Regarding accuracy and jitter, better results on both parameters were detected inside the recommended working area for both devices (Table 2). Similar values were obtained for accuracy and jitter at both heights for both devices, except for the HTC Vive's accuracy, which was better at lower heights. Both devices showed comparable performance at sitting height, both within and beyond the recommended working area. At standing height, however, the HTC Vive presented worse accuracy and jitter.

The working range of the Oculus Rift's tracking system was $4.25 \mathrm{~m}$ at both heights, starting at $0.25 \mathrm{~m}$ away from the camera. The HTC Vive presented higher working ranges at both heights, up to $6.75 \mathrm{~m}$ at a sitting height and $7 \mathrm{~m}$ at a standing height.

\section{Discussion}

This research involved comparing the working area, working range, accuracy, and jitter in a room-size setting for two latest-generation HMDs: the Oculus Rift and the HTC Vive. The HTC Vive provided a potential working area of nearly $25 \mathrm{~m}^{2}$, twice as large as that of the Oculus Rift, and a working
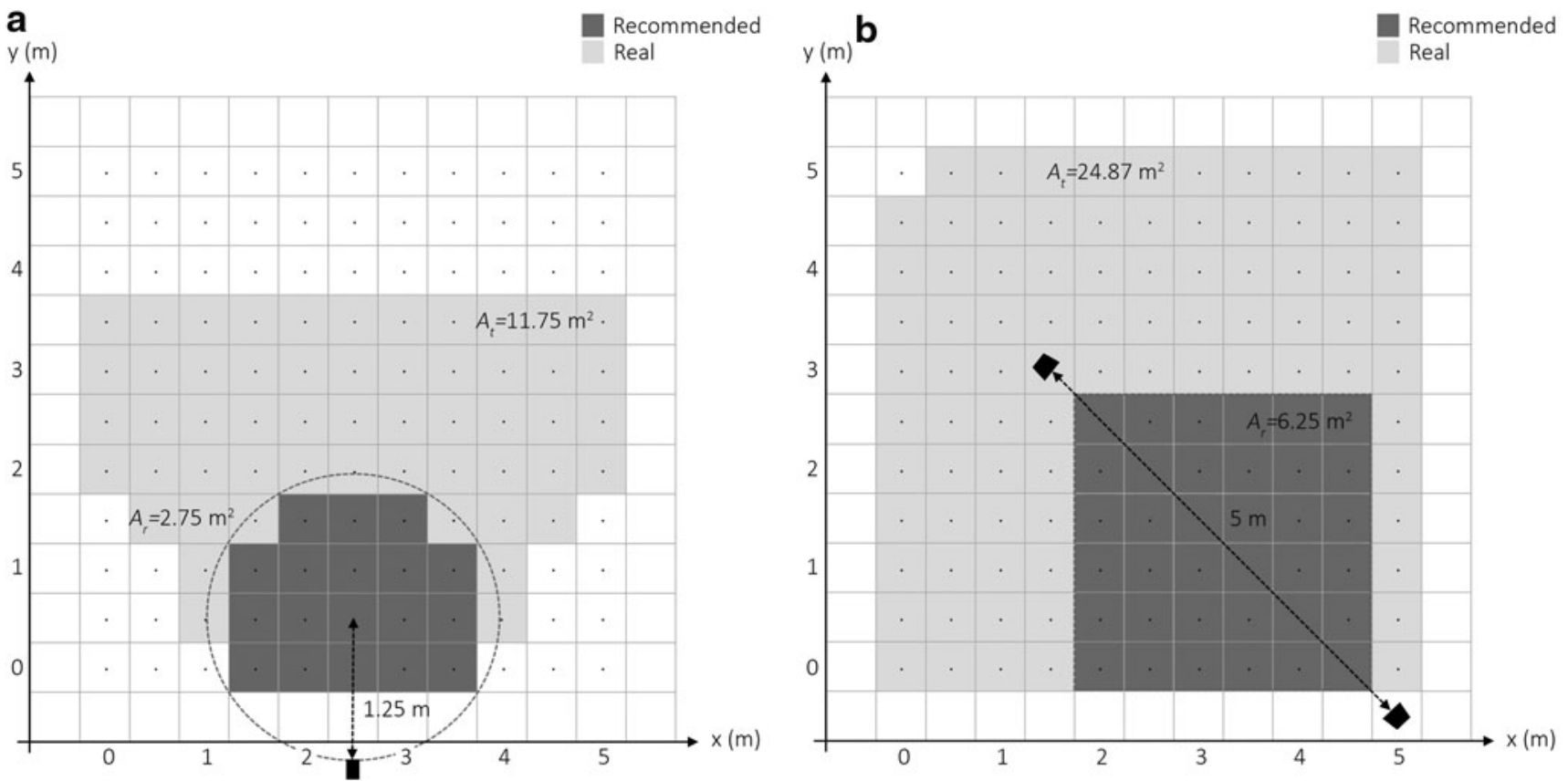

FIG. 3. Comparison of the recommended and real working area registered for (a) the Oculus Rift and (b) the HTC Vive. range of $7 \mathrm{~m}$. Both devices showed excellent performance in terms of accuracy and jitter.

Both the Oculus Rift and the HTC Vive provided a working area that largely exceeds the spatial requirements of most nonimmersive VR-based serious games, exergames, and motor rehabilitation applications. ${ }^{15,21-25}$ Moreover, the working area of the head tracking of the latest-generation HMDs potentially enables navigation in life-size virtual environments, such as kitchens, ${ }^{26,27}$ supermarket aisles, ${ }^{28,29}$ or streets, ${ }^{30,31}$ which have been traditionally used in rehabilitation and health-related applications due to their functional implications. Navigation within these virtual environments (joysticks, keyboards, etc.), but the head tracking of the new HMDs could overcome this abstraction and enable human navigation and exploration, thus enhancing the ecological validity of the tasks and facilitating skill transference. ${ }^{18}$ This capability could also be combined with other techniques to "virtually" enlarge the walking area, such as dynamic layout Navigation could be especially feasible with the HTC Vive, whose working area is twice as large as the area provided by the Oculus Rift. In addition, the HTC Vive has a $360^{\circ}$ field of view, which enables natural exploration in all spatial directions. The HTC Vive's use of two base stations, in contrast to only one camera, and its specific tracking mechanism (see Instrumentation section) could explain this difference. The addition of more cameras to the Oculus Rift setting, which was enabled from version 1.6 of the runtime package, together with a dramatic increase of the working area, could improve the results presented here. It is important to highlight that these cameras are not currently included in the standard bundle and should be acquired additionally.

The HTC Vive's larger working area could also explain its worse accuracy for the standing height when considering the whole area. This fact could evidence the distance has been commonly facilitated by external peripherals generation, ${ }^{32}$ redirect walking, ${ }^{33}$ or motion compression. ${ }^{34}$ 
Table 2. Accuracy and JitTer Data

\begin{tabular}{|c|c|c|c|c|c|c|}
\hline & \multicolumn{2}{|c|}{ On the floor $(0 \mathrm{~m})$} & \multicolumn{2}{|c|}{ Sitting position $(1.3 \mathrm{~m})$} & \multicolumn{2}{|c|}{ Standing position $(1.7 \mathrm{~m})$} \\
\hline & Oculus Rift & HTC Vive & Oculus Rift & HTC Vive & Oculus Rift & HTC Vive \\
\hline \multicolumn{7}{|c|}{ Accuracy $(\mathrm{cm})$} \\
\hline Inside & - & $0.58 \pm 0.45$ & $0.76 \pm 0.53$ & $0.75 \pm 0.69$ & $0.61 \pm 0.55$ & $1.22 \pm 1.18$ \\
\hline Outside & - & $0.85 \pm 0.84$ & $1.03 \pm 0.74$ & $0.93 \pm 0.72$ & $0.92 \pm 0.75$ & $1.49 \pm 1.38$ \\
\hline \multicolumn{7}{|l|}{ Jitter (mm) } \\
\hline Inside & - & $0.19 \pm 0.26$ & $0.12 \pm 0.18$ & $0.23 \pm 0.33$ & $0.13 \pm 0.46$ & $0.31 \pm 0.56$ \\
\hline Outside & - & $0.27 \pm 0.37$ & $0.25 \pm 0.48$ & $0.32 \pm 0.44$ & $0.36 \pm 0.54$ & $0.44 \pm 0.99$ \\
\hline
\end{tabular}

Mean values of the accuracy and jitter of both devices at different heights inside and outside the working area. Data are expressed in terms of mean and standard deviation.

dependence of this variable, which seems to be greater for the Oculus Rift. However, the performance of both tracking systems, even in the worst case, should be highlighted and put into context. First, the Oculus Rift's head tracking accuracy, with average values ranging from 0.6 to $1.2 \mathrm{~cm}$, represented $<0.35 \%$ of its working range. In turn, the HTC Vive's head tracking accuracy, with average values ranging from 0.9 to $1.5 \mathrm{~cm}$, represented $<0.21 \%$ of its working range. Second, both HMDs' head tracking accuracies proved to be smaller than those of other optical cameras and electromagnetic systems used in VR-based serious games and rehabilitation. ${ }^{20}$ Third, jitter was even more noteworthy, since the HMDs included for analysis showed only $10 \%$ of the jitter provided by motion tracking systems commonly used in VR. ${ }^{20}$ Fourth, although the accuracy of the HMDs' head tracking proved to be up to three times worse than markerbased solutions, ${ }^{17}$ jitter was up to three times smaller and does not require multiple markers to be attached to the ceiling, which makes set up easier and more affordable. Finally, and most importantly, results should be referred to human perception. During passive observation of static visual information in a big LCD screen, head movements have been reported to be up to $2 \mathrm{~cm}$ in average, with peaks of 3$4 \mathrm{~cm},{ }^{35}$ which is $45-90$ times the jitter of the HMDs' tracking. Interestingly, head sway under similar conditions was nearly two orders of magnitude higher than the jitter provided by the HMDs' head tracking, which could make it virtually imperceptible and prevent cybersickness or other deleterious effects.

\section{Conclusion}

The experimental results for working area, accuracy, and jitter of the latest-generation HMDs' tracking support the use of these devices not only as a feasible alternative for VRbased health applications, such as serious games, exergames, and motor rehabilitation systems, but also to enable the navigation and exploration of real-life size virtual environments, without any remarkable adverse effects.

\section{Acknowledgments}

This study was funded, in part, by Ministerio de Economía y Competitividad of Spain (Project NeuroVR, TIN201344741-R, and Grant No. BES-2014-068218) and by Universitat Politècnica de València (Grant Nos. PAID-10-14 and PAID-10-16).

\section{Author Disclosure Statement}

No competing financial interests exist.

\section{References}

1. Bermúdez i Badia S, Fluet GG, Llorens R, Deutsch JE. Virtual reality for sensorimotor rehabilitation post stroke: Design principles and evidence. In: Neurorehabilitation Technology, 2nd ed. Edited by DJ Reinkensmeyer and V Dietz. Springer, 2016, pp. 573-603.

2. Slater M. Measuring presence: A response to the Witmer and Singer presence questionnaire. Presence Teleoperators Virtual Environ 1999;8:1-13.

3. Baños RM, Botella C, Alcañiz M, et al. Immersion and emotion: Their impact on the sense of presence. Cyberpsychol Behav 2004;7:734-741.

4. Malcolm GL, Groen IIA, Baker CI. Making sense of realworld scenes. Trends Cogn Sci 2016;20:843-856.

5. Sereno MI, Dale AM, Reppas JB, et al. Borders of multiple visual areas in humans revealed by functional magnetic resonance imaging. Science (80-) 1995;268:889-893.

6. Cruz-Neira C, Leigh J, Papka M, et al. Scientists in wonderland: A report on visualization applications in the CAVE virtual reality environment. Proceedings of the IEEE 1993 Symposium on Research Frontiers in Virtual Reality, 1993, San Jose, CA, 1993, pp. 59-66.

7. Sutherland IE. A head-mounted three-dimensional display. Proceedings of the AFIPS'68 (Fall, Part I), San Francisco, CA, 1968, pp. 757-764.

8. Rizzo AS, Kim GJ. A SWOT analysis of the field of virtual reality rehabilitation and therapy. Presence Teleoperators Virtual Environ 2005;14:119-146.

9. Bellini H, Chen W, Sugiyama M, et al. Virtual \& Augmented Reality: Understanding the race for the next computing platform. Profiles Innov 2016:1-30.

10. Deb S, Carruth DW, Sween R, et al. Efficacy of virtual reality in pedestrian safety research. Appl Ergon 2017;65:449-460.

11. Jean dit Gautier E, Bot-Robin V, Libessart A, et al. Design of a serious game for handling obstetrical emergencies. JMIR Serious Games 2016;4:e21.

12. Nunnerley J, Gupta S, Snell D, King M. Training wheelchair navigation in immersive virtual environments for patients with spinal cord injury - end-user input to design an effective system. Disabil Rehabil Assist Technol 2017;12:417-423.

13. Kim A, Darakjian N, Finley JM. Walking in fully immersive virtual environments: An evaluation of potential adverse effects in older adults and individuals with Parkinson's disease. J Neuroeng Rehabil 2017;14:16. 
14. Colomer C, Llorens R, Noé E, et al. Effect of a mixed reality-based intervention on arm, hand, and finger function on chronic stroke. J Neuroeng Rehabil 2016;13:45.

15. Lloréns R, Gil-Gómez J-A, Alcañiz M, et al. Improvement in balance using a virtual reality-based stepping exercise: A randomized controlled trial involving individuals with chronic stroke. Clin Rehabil 2015;29:261-268.

16. Lloréns R, Noé E, Colomer C, Alcañiz M. Effectiveness, usability, and cost-benefit of a virtual reality-based telerehabilitation program for balance recovery after stroke: A randomized controlled trial. Arch Phys Med Rehabil 2015;96:418.e2-425.e2.

17. Borrego A, Latorre J, Llorens R, et al. Feasibility of a walking virtual reality system for rehabilitation: Objective and subjective parameters. J Neuroeng Rehabil 2016;13:68.

18. Rauter G, Sigrist R, Koch C, et al. Transfer of complex skill learning from virtual to real rowing. PLoS One 2013;8:e82145.

19. Davies A. Oculus Rift Vs. HTC Vive Vs. PlayStation VR. tomshardware.co.uk. 2016. www.tomshardware.co.uk/viverift-playstation-vr-comparison,review-33556-3.html (accessed December 28, 2016).

20. Lloréns R, Noé E, Naranjo V, et al. Tracking systems for virtual rehabilitation: Objective performance vs. subjective experience. A practical scenario. Sensors (Switzerland) 2015;15:6586-6606.

21. McEwen D, Taillon-Hobson A, Bilodeau M, et al. Virtual reality exercise improves mobility after stroke: An inpatient randomized controlled trial. Stroke 2014;45:1853-1855.

22. Rand D, Kizony R, Weiss P (Tamar) L. The Sony PlayStation II EyeToy: Low-cost virtual reality for use in rehabilitation. J Neurol Phys Ther 2008;32:155-163.

23. Sin $\mathrm{H}$, Lee $\mathrm{G}$. Additional virtual reality training using Xbox Kinect in stroke survivors with hemiplegia. Am J Phys Med Rehabil 2013;92:871-880.

24. Street TD, Lacey SJ, Langdon RR. Gaming your way to health: A systematic review of exergaming programs to increase health and exercise behaviors in adults. Games Health J 2017;6:136-146.

25. Lin J, Kelleher CL, Engsberg JR. Developing home-based virtual reality therapy interventions. Games Health J 2013; 2:34-38.

26. Zhang L, Abreu BC, Masel B, et al. Virtual reality in the assessment of selected cognitive function after brain injury. Am J Phys Med Rehabil 2001;80:597-604; quiz 605.
27. Klinger E, Cao X, Douguet AS, Fuchs P. Designing an ecological and adaptable virtual task in the context of executive functions. Annu Rev CyberTherapy Telemed 2009; 7:248-252.

28. Sorita E, Joseph PA, N'kaoua B, et al. Performance analysis of adults with acquired brain injury making errands in a virtual supermarket. Ann Phys Rehabil Med 2014;57:e85.

29. Okahashi S, Seki K, Nagano A, et al. A virtual shopping test for realistic assessment of cognitive function. J Neuroeng Rehabil 2013;10:59.

30. Navarro M-D, Lloréns R, Noé E, et al. Validation of a lowcost virtual reality system for training street-crossing. A comparative study in healthy, neglected and non-neglected stroke individuals. Neuropsychol Rehabil 2013;23:597618.

31. Devos H, Akinwuntan AE, Nieuwboer A, et al. Comparison of the effect of two driving retraining programs on on-road performance after stroke. Neurorehabil Neural Repair 2009; 23:699-705.

32. Vasylevska K, Kaufmann H, Bolas M, Suma EA. Flexible spaces: Dynamic layout generation for infinite walking in virtual environments. 2013 IEEE Symposium on 3D User Interfaces (3DUI). IEEE, Orlando, FL, 2013, pp. 39-42.

33. Razzaque S, Kohn Z, Whitton MC. Redirected Walking. Proc EUROGRAPHICS 2001:289-294.

34. Nitzsche N, Hanebeck UD, Schmidt G. Motion compression for telepresent walking in large target environments. Presence Teleoperators Virtual Environ 2004;13:44-60.

35. Ciria LF, Muñoz MA, Gea J, et al. Head movement measurement: An alternative method for posturography studies. Gait Posture 2017;52:100-106.

Address correspondence to: Roberto Llorens, PhD Neurorehabilitation and Brain Research Group Instituto de Investigación e Innovación en Bioingeniería Universitat Politècnica de València Camino de Vera s/n Valencia 46011 Spain

E-mail: rllorens@i3b.upv.es 